\title{
Data integration of cost materials needs through a service oriented architecture (study case: $p t x$ garment ungaran)
}

\author{
Erwien Christianto $^{1 *}$, Wiranto Herry Utomo ${ }^{2}$, Wiwin Sulistyo ${ }^{1}$ \\ ${ }^{1}$ Faculty of Information Technology Satya Wacana Christian University, Diponegoro St 52-60, Salatiga 50711 Central Java, Indonesia \\ ${ }^{2}$ Magister of Information Systems Satya Wacana Christian University, Diponegoro St 52-60, Salatiga 50711 Central Java, Indonesia \\ *Corresponding author E-mail:erwinchristiant@gmail.com
}

Copyright (1) 2015 Erwien Christianto et al. This is an open access article distributed under the Creative Commons Attribution License, which permits unrestricted use, distribution, and reproduction in any medium, provided the original work is properly cited.

\begin{abstract}
Data processing is an important part of a garment company. With increasingly complex and developing a garment company, data processing and integration became a very important requirement. The need for data integration in determining the cost of materials becomes a very important part in the garment industry. Data distribution or dissemination from one to another section gives results in data duplication, so that it may cause the data to be inconsistent. In addition, the efficiency of the process in determining the cost of materials is highly needed to achieve the selling price determination target. Nowadays, there is web-based technology, which is capable of handle data integration service, called SOA (Service Oriented Architecture). Business processes (work flows) involving the supplier need to go back to the supplier with the output price that has been determined by the system, can be integrated with Web service with concentration of BPELSOA. By utilizing the SOA technology then data processing and integration problems that occurred in the garment industry could be made into an integrated information system, so that the problems in the garment industry can be solved.
\end{abstract}

Keywords:Soa; Ws; Bpel; Integration; Cost of Materials Information Systems.

\section{Introduction}

Nowadays, garment industry has several important parts that interact one another and divided into several departments or separate divisions led by a department head or manager. Some garment industries in Indonesia, each has its own terms and names according to the functions such as marketing, merchandising, production planning, fabric and other parts in the company.

Each department such as marketing and PPIC have output as a determinant of the cost of materials, PDF department as the pattern maker, GGT as consumption sheet determinant, IE as a time studies a determinant, all of which have relevance in determining the cost of materials. Problems that often arise in the process of determining the cost are duplication between the current data information dissemination distribution to each department and inefficiency due to the manual data processing, which cause data accumulation. Information delivery problem between departments can take advantage of Service Oriented Architecture which is the right solution to address multiple data flow which is very helpful to solve those problems.

SOA offers a good infrastructure with integration capability that have been managed well, Service Oriented Architecture (SOA) is a software-development architecture technology with service approach, which allow the data or information connection and exchange between departments become easy. It's loosely coupled (low level of dependence between components). Highly interoperable (easy to operate), reusable, and interoperability (can communicate across platforms) characteristics make SOA a reliable system in the information development and integration [1].

The implementation of SOA using Web Service is an appropriate thing, because this technology can represent a service in SOA and by the utilization of this web service, the new potential of SOA can be raised. So Web Service is suitable for implementing concepts and properties of SOA, and Enterprise Service Bus (ESB) provides the infrastructure to 
implement the concept of Service Oriented Architecture (SOA). ESB is a new technology trend for SOA integration architecture. Communication between departments in garment industry and the supplier of the service is not performed by emailing anymore but through a service bus. Therefore, the implementation of SOA by using Web Service technology can be performed by building orchestration based on services, which are appropriate for the needs of the business processes in the $\mathrm{X}$ garment, Ltd Ungaran as a case study in this study.

\section{Literature review}

There are several previous studies as comparison references or to distinguish the research findings conducted: Another study was also conducted on the development of software, called software as a Service with an SOA service-oriented architecture where there is a centralized application that is managed by a software service provider, which can handle multiple different company data. With this concept than data storage and transactions on inventory applications are separated on different schema, in order to use the application by more than one company exclusively [2].

Another study conducted in a large phone company T-Mobile which applied CRM with SOA to align different business processes from the front to back. [3]

Furthermore, a study on Software integration (Enterprise Resource Planning) ERP with (Service Oriented Architecture) SOA. The concept of ESB supported the implementation of Service Oriented Architecture paradigm. Interaction between service components was performed through ESB mediator. This would bring loose-coupling characteristic on the interaction between services and simplify management in a distributed system [4].

\subsection{SOA}

[5] SOA is a way of designing application by using the existing components or services or in other words, an application which is built on a modular basis. Actually, this modular approach is not a new thing. Contemporary programming techniques such as object-oriented programming, has put a modular approach forward in the development of applications. However, what makes SOA different is a component or service which is constructed and interact one another freely and loose (loose coupled).

[5] With the loose-coupled characteristic, a service can be invoked by another program / service without the need to pay on the location of the invoked service and what platform / technology is being used by the service. Loose coupling is very important for SOA because a service call by other services can be performed at a run-time.

[5] For example, a core banking application provides a Fund Transfer service, then the applications such as banking, treasury, payment gateway, ATM switching and others can invoke the Fund transfer service without the need to search where the Fund Transfer is located within the network and invoke technique to be used. This contrasts with the tight coupling approach in which each banking application must have a Fund Transfer function in it so that it will be difficult and costly/need great resources to change the logic from Fund Transfer to new business requirements in every application.

[5] Other characteristics are that service in SOA is constructed on two things: Service Interface and Service Implementation.

1) Service Interface expresses how the service can be called as an input / output parameter and also its location. For example, service interface for the Customer Lookup expresses a variety of ways to obtain information about a customer (from the customer id or name, and so on) and the structure of customer data that is returned. Service Implementation is how the logic of the Customer Lookup service is run.

2) Service implementation is strongly correlated with the programming technology used. SOA does not need to care about how a service is implemented. Whether written in Java or COBOL, the most important thing is how the service can be called and provides information in accordance with the Service Interface.

3) Business oriented. In a sense, each service is defined to do a particular business activity, e.g. Customer Lookup, Fund Transfer, Check Inventory, and so on. It is inevitable also that latest SOA success is driven by the high acceptance of web services technology among application developers. Although the idea of SOA has been around before the existence of web services, web services and SOA have now become a synergy, and even some people consider that by using the web service then they have implemented SOA [6].

Figure 1 is a picture of SOA architecture when it was, firstly, introduced. SOA has three main parts: the service requestor, the service provider, and the service registry. Service registry and service provider are correlated with Web Service Definition Language (WSDL) as a tool to define the service provided. While the link between service provider and service requestor is Simple Object Access Protocol (SOAP), which is the standard for sending messages from the two services. Then in service registry and service requestor there are Universal Description Discovery and Integration (UUDI) which are the standardization of service registry. 


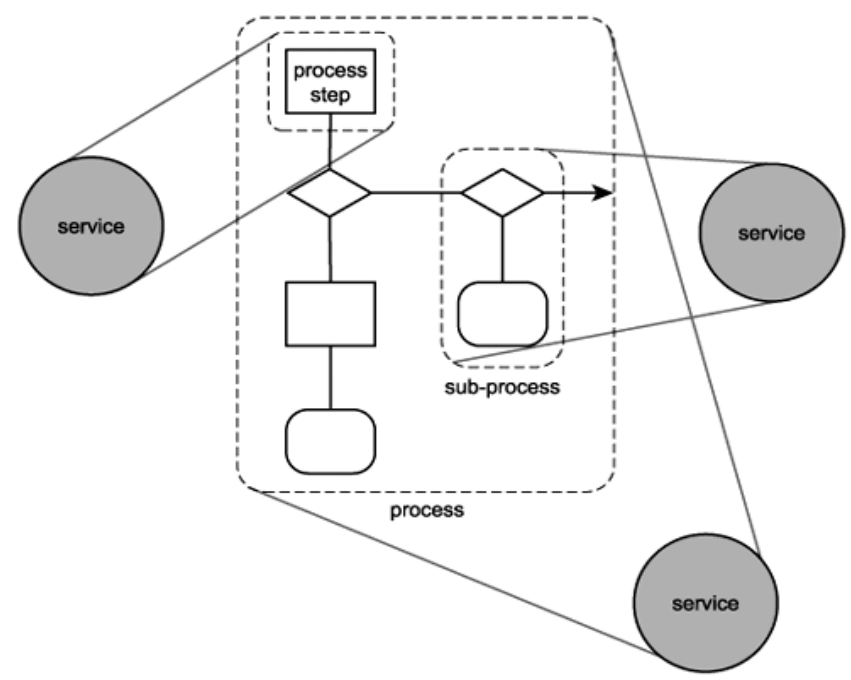

Fig. 1:Service Architecture in SOA

According to Thomas Erl, service can be divided into several smaller parts. However, the scope for each service can be varied as shown in Figure1. The figure shows that each service has a different service, one service handles a small number of problems called subprocesses, and other service handles almost all problems encountered. This makes it easy to add multiple services without changing the architecture that has been used [7].

\subsection{ESB(enterprise service bus)}

ESB is an infrastructure for integrating applications and services. ESB strengthens SOA by reducing the number, size, and complexity of interfaces, applications and services. ESB is used to connect to multiple IT resources. ESB must be flexible to combine and re-install the components according to changing business needs. ESB performs component connection, which is loosely bound, thus providing the ability to integrate a system into SOA and deploy gradually [8].

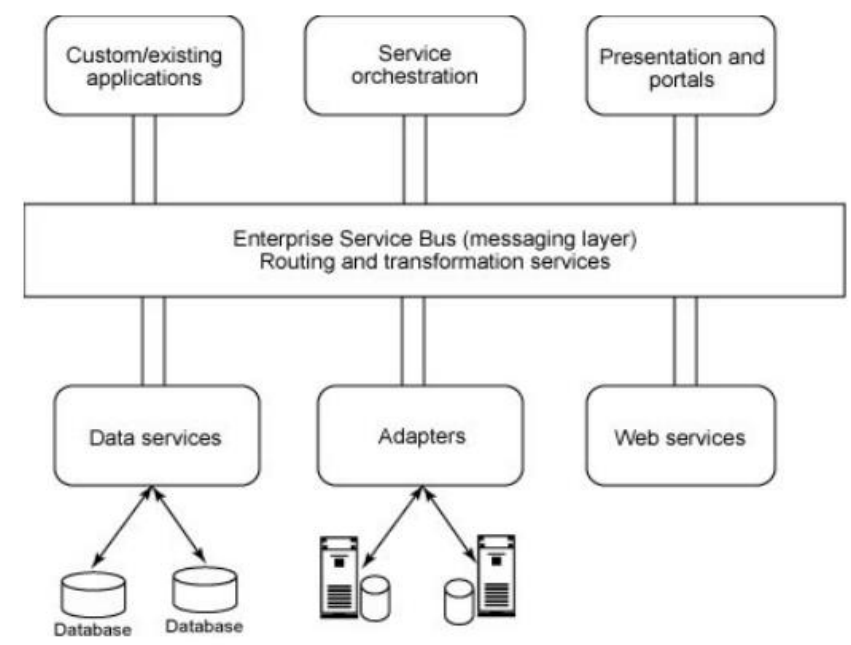

Fig. 2:ESB[9]

\subsection{Web service}

Web service is a software system designed to support interoperable interaction between machines over a network. Although the concepts that make up, SOA are exist before the emerging of web service, web service has an important role in SOA. The rapid development of a web service led to the existence of web service extensions that extend the Web service functions themselves.

This is because web services are built on protocols that are well-known and have independent platforms, such as HTTP, XML, UDDI, and WSDL. SOA uses those protocols as key components since that protocol provide services that can be discovered and used dynamically. SOA provides a service that has to interface contract that is platform independent, provided by XML. SOA emphasizes interoperability, and it is provided by HTTP. These reasons make the web services become the heart of SOA. 
Here's an explanation of the processes that occur in the web service:

a) The service finding process (find). This process is performed by the service consumer to the server who has a list of services that can be used as well as how to use it.

b) The service utilization processes (bind and invoke). This process is performed when an agreement between service consumer and service provider has registered in the registry server.

c) Register Process. This process is performed by the service provider to register services that can be used by the service consumer on the registry server.

d) Service contract. This section is a service interface that defines the components contained in a service. Service contract is represented in the form of WSDL. WSDL is a language description that can be understood by a human and a computer machine. WSDL is written using XML syntax and is used as an interface to exchange messages in a service. A web service application can not immediately be known how it is used. Therefore, this application must provide a description of the service. For this description, the web service also has an open standard that is WSDL (Web Service Description Language) [10] [11].

\section{Methodology}

(SOA) is a software engineering model integrated with business processes in an information system was established as standard component modules and can be reused by other business processes.

In figure 3, according to Thomas Erl, Service was divided into varied sections. Each service handled the business process to process within. This was performed to facilitate the addition of a new system without changing the architecture of the existing business processes.

Thomas Erl summarized the steps in building service design of the business processes [12] [13] [14] Bieberstein.

The first step is integration map design. This step was designed to determine the needs of the service process by sequentially describe the overall business process, while the service candidate who will be made based on the business process can be obtained from it.

The second step was process service interface design. After finding the definition of the service process, the process using the tools in the WSDL web service will be automatically public.

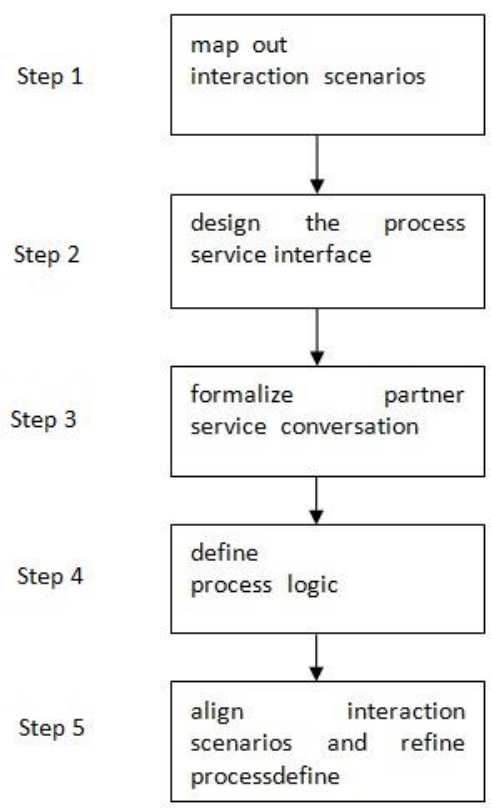

Fig. 3:Stages by Thomas ERL

The third step was formalizing the conversation services partners. This step would combine the services that have been made previously by interactions with other services. The fourth step was determining the logic process. This step would evaluate the service logic plot that has been made with the existing business processes.

The fifth step was aligning scenario interaction and improving the process. This step is optional. By doing scenario interaction in step 1, what make a service has been served by a scenario and be turned away from the business privacy component issue of distributed applications. [15] In implementations which are built based on Erl method, through the needs design of the service process up to the second step by defining service design of the logic process interface until the application. 


\section{Running system analysis}

Determining the starting point for the implementation of SOA in the production information systems of manufacturing company is a very important part in determining the environmental strategy issues that will be performed. Problem Environment to be executed is to meet the needs of the organization, a support unit and the flow between departments, especially marketing relationship with customers and marketing with interrelated parts in it to be integrated into the existing information systems in X garment company, Ltd. Ungaran. Business process flow uses the UML (Unified Modeling Language) design method.

\subsection{Order needs business process}

Description:

1) The marketing department gives the layout to the PPIC (Production Planning and Inventory Control) with a purpose to make costing.

Based on the layout:

2) PPIC (Production Planning and Inventory Control) decides how many meters/yards do 1 piece of dress takes fabric.

3) PPIC (Production Planning and Inventory Control) decides how much time to make one shirt (based on the Tech Pack).

4) Based on the calculation information (SMV\& Consumption fabric) and PPIC (Production Planning and Inventory Control) to determine Costing.

Based on the business process above, integration built is limited to the basic material needs those are descriptions 1-4 of IC/Marketing department, PPIC, GGT and IE that can be described as the flow of raw materials cost needs in figure 4. Therefore, the cost of raw materials will be calculated to be used as the basis for calculating the selling price for one shirt and given to the supplier before any agreement for PO (Purchase Order).

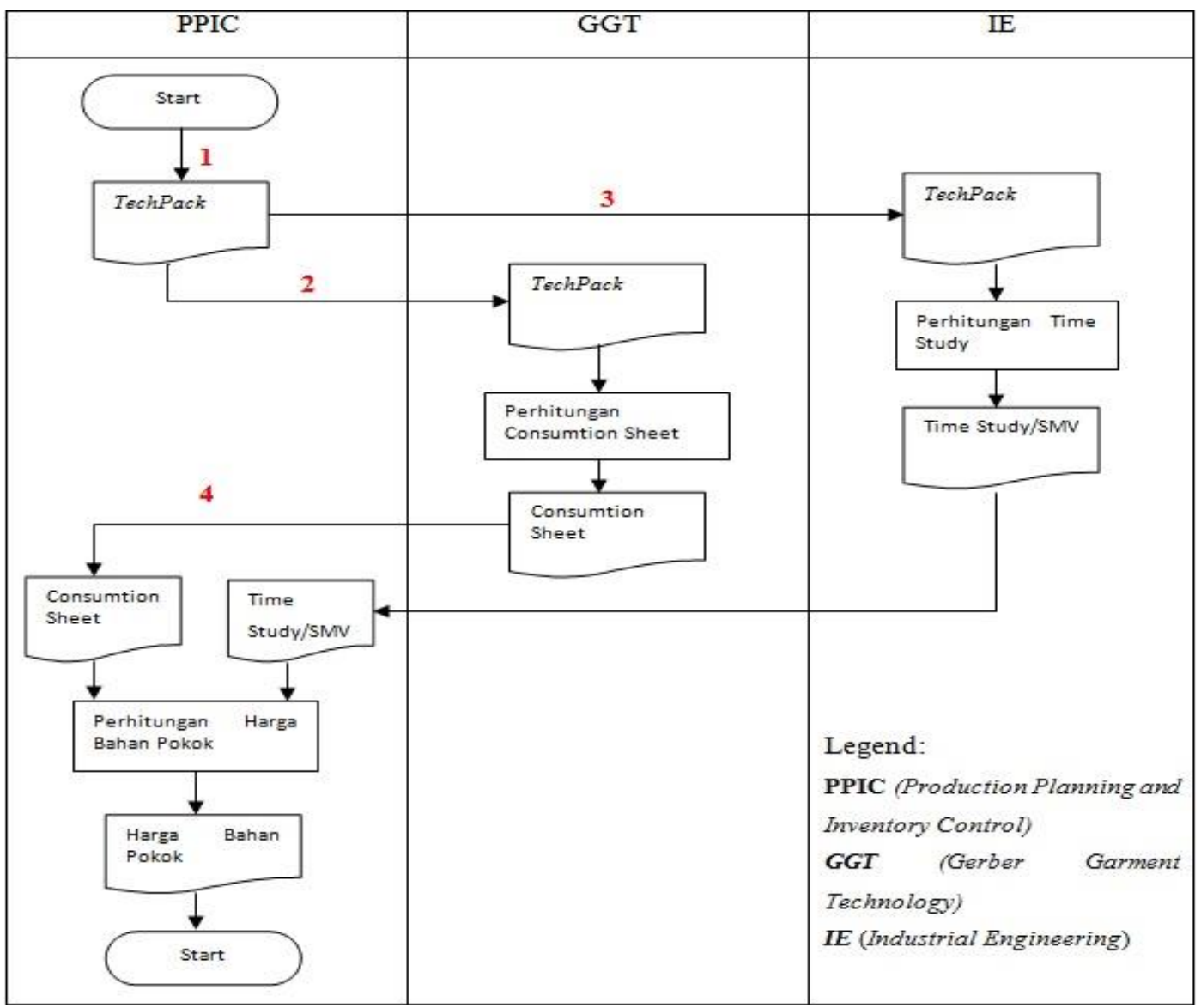

Fig. 4:Flow of Raw Materials Cost Needs

In determining the cost of materials, Part PPIC will send sales price consists of the price of capital and profits and it will be given to buyers .For shown can determine the value of the Cost of Materials calculations required at each part generated. The result will be: 
1) IE

SMV (Standart Minute Value)

SMV $=($ Body + Pintuck + Button $)$

2) GGT

a) Digitizer:

- $\quad$ Size S \& XL: Yards, inches

- $\quad$ Size M \& L: Yards, inches

b) GGT

Total $=\operatorname{Size}(X s+S+M+L+X L)$

T.Yards $S, X L=\left(\left(\frac{\text { Inches } X L}{36}\right)+\operatorname{Yards} X L\right) \times$ SizeS

T.Yards $M, L=\left(\left(\frac{\text { Inches } M}{36}\right)+\right.$ Yards $\left.M\right) \times$ SizeL

$E x \% S, X L=((0,03 \times T . Y a r d s S)+T . Y a r d s S)$

$E x \% M, L=((0,03 \times T . Y a r d s M)+T . Y a r d s M)$

Tot $\quad=(E x \% S, X L+E x \% M, L)$

$\left(\right.$ Cons $\left./{ }_{P c}\right)=\frac{\text { Tot }}{\text { Total }}$

3) PPIC:

$($ Value $/$ USS $)=\left[\right.$ Rate + Cons $\left./ P_{C}+L / I\right]$

L: Local (3\%)

I: Import (5\%)

\subsection{Results of integration design}

Phase 1 includes the design of this integration is built in the scope that contents parts in the company. Those are: PPIC (Production Planning and Inventory Control) as a determinant of raw material cost after receiving information from IE and GGT, IE (Industrial Engineering) is a part that determines time produced to make one dress. GGT (Garber Garment Technology) is a part that accounts and produces fabric consumption for every one dress; PDF (Product Development Facilities) is the first part in the production process that has the main job of cutting material. However, the scope is limited to the PPIC, GGT and IE parts.

Here is the design of architectural design that will be built in the study on integration of material cost needs business process with web service technology and SOA.

\subsection{Use case diagram}

Each step in use case is an element in the interaction between the actor and the system. Each step should be a simple statement that clearly shows who is running the step. Use case diagram shows three aspects of the system: actor, use case and system or sub-system boundary. Actor represents people role, other systems or tools when communicating with a use case. Use case is an abstraction of the interaction between the system and the actor. Use Case image consists of four actors those are IC, PIC, IE and GGT parts. These sections have the right to view, input and edit data. 


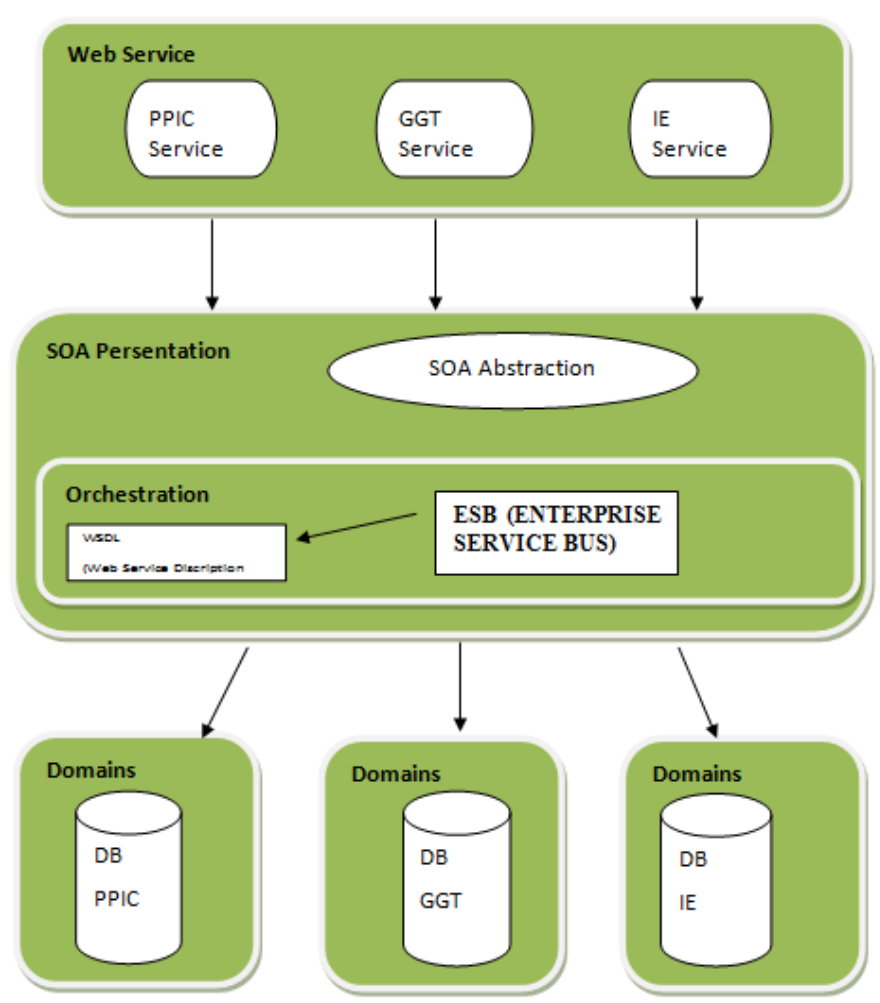

Fig. 5:Architectural Design of Material Cost Needs

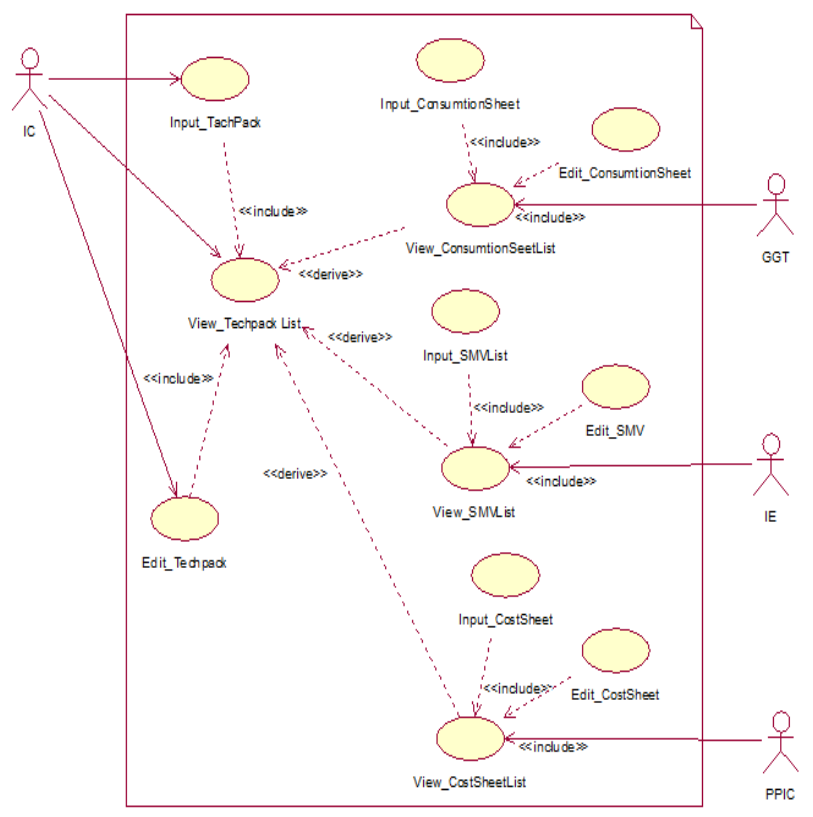

Fig. 6: Use Case Diagram

\subsection{Activity diagram}

Activity diagram is a special state diagram; that the activity diagram does not describe the internal behavior of a system (and the interaction between the subsystems) exactly, but rather describes the activity processes and pathways of the above level generally. Activity diagram is a combination of several techniques. Those are: event diagrams by Jim Odell, SDL state modeling techniques, work-flow modeling, and Petri nets. These diagrams are useful in connection with the workflow and describe the activities carried out together in parallel. Thus the activity diagram describes the stages of various activities that support two or more conditions and activities in parallel.

Here is the activity diagram flowchart and in the flow of information that results in the cost of materials in the relevant sections. It can be seen from the activity diagram in Figure 7. 


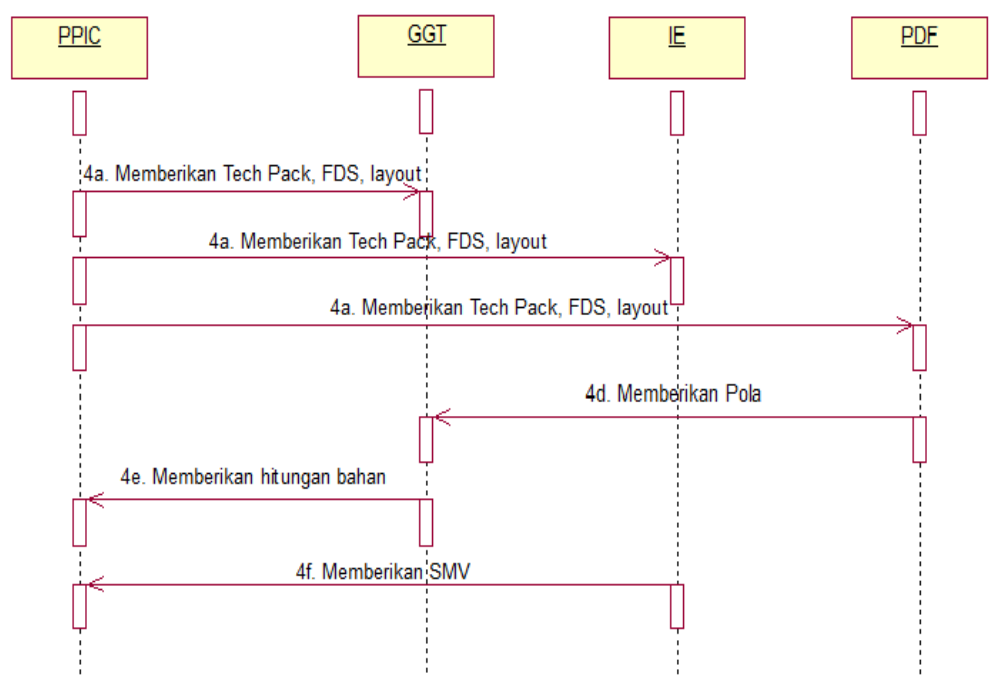

Fig. 7: Activity Diagrams

\subsection{Class diagram}

Class is a specification that if it is made an instant will produce an object as the core of development and object-oriented design. Class describes the state (attributes/properties) of a system, while offering services to manipulate the situation (method/function). Class diagram describes the structure and description of classes, packages and objects and their relationships to each other such as containment, inheritance, associations, and others.

Each section has a required parameter in accordance with the needs of the data. In IC part, has parameter inputted with the Techpack document name, and in GGT part, there is a parameter for the fabric needs with Consumption Sheet documents, IE part has a parameter for the time/Time Study needs of one dress and PPIC part has a parameter needed to generate the cost of materials. We can see it from the class diagram in Figure 8.

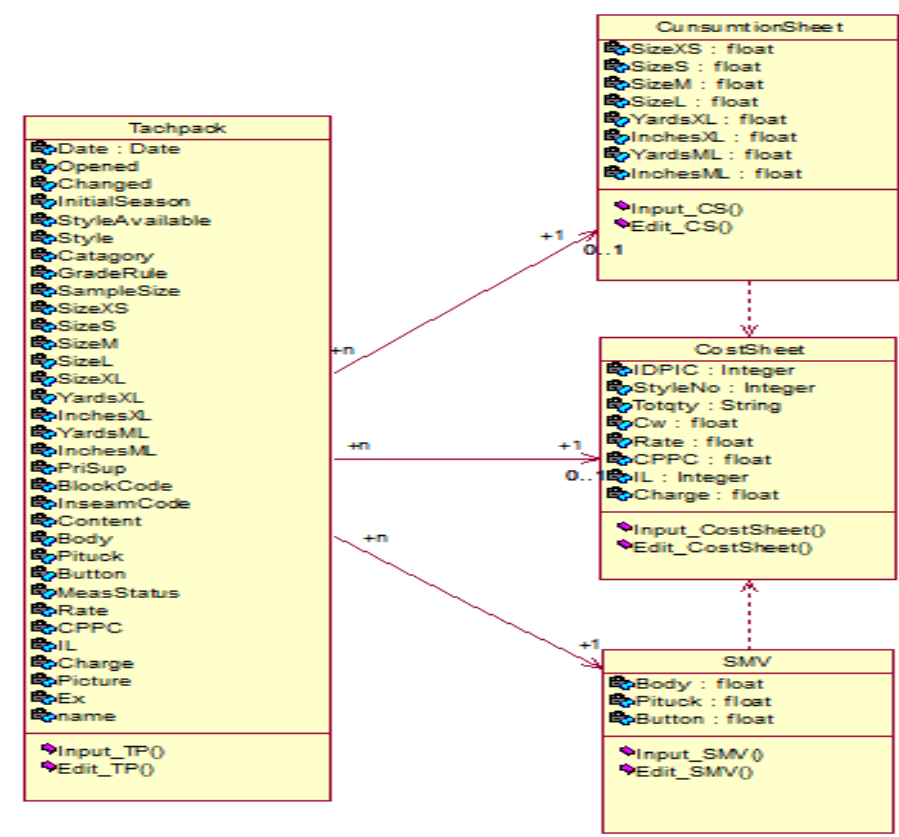

Fig. 8: Class Diagram

\subsection{Web service implementation}

Phase 2 building services with a web service describe the flow of business processes from each section. Service that is built based on the parameters required for each section directly with application software using XML-based messages over internet protocol. Messages delivered via XML will be displayed by SOAP (Simple Object Access Protocol) respond and requests element. An example of a web service that is used to produce fabric needs for one dress can be seen from one sample of the web service in the GGT part in Figure 9. 


\begin{tabular}{|l|l|}
\hline float & 4 \\
\hline float & 20.655 \\
\hline float & 4 \\
\hline float & 20.746 \\
\hline java.lang.String & 0 \\
\hline Method returned \\
\hline double : "2.3563490831163194" \\
\hline
\end{tabular}

\section{SOAP Request}

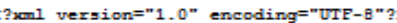

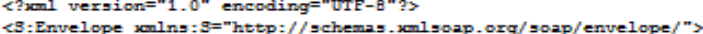

$\langle s:$ Header/>

Lns2: Khohn wellns:ns 2="http://WGGGT/">

$<$ SiveX3>0.0</3iveXS>

$<$ SireS>2400.0</BireS>
$\langle$ SireM $>4800.0</$ BireM>

$\langle$ SireL>4800,0</SireL>

<BiveXL>2400.0</3iveXL)

$<$ YyardsBXL>4.0</yards SXL)

<Inches SXL>20. $655<$ / InchessXL)

$<$ YardaML $>4.0</$ Y 2 rdaML.

<InchesML > $20.746</$ InchesML>

\section{Body>}

SOAP Response

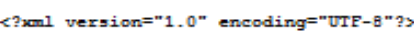

<3:Envelope xemlns: $\$=" h t t p: / /$ schemas. xmlsoap.org/soap/envelope/">

$\langle 3:$ Body $>$

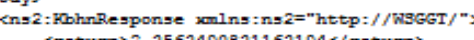

S2:

$</ 3:$ Envelopes

Fig. 9:Web Service Result of GGT Part

\subsection{Implementation with ESB}

Implementation of Business Processes by using BPEL 2.0 on Glassfish ESB can be seen in Figure 10. From a database built using MySQL database, we can see BPEL business searching process. In the business process, the process is initiated by the service user (Trigger Searching BP) who makes a request to Searching BP. Service users are implemented with WSDL that is sent by using SOAP, while the service providers in the form of databases are wrapped in WSDL.

Based on the business process with BPEL implementation can be viewed between Link partners of the input in IC to be intercepted by the parts that need it. GGT part captures some parameters given by IC with service consumption sheet. IE part captures some parameters given by IC with SMV service and PPIC part that captures some parameters given by with service Cost Sheet. The output results cost of materials will be shown in the IC.

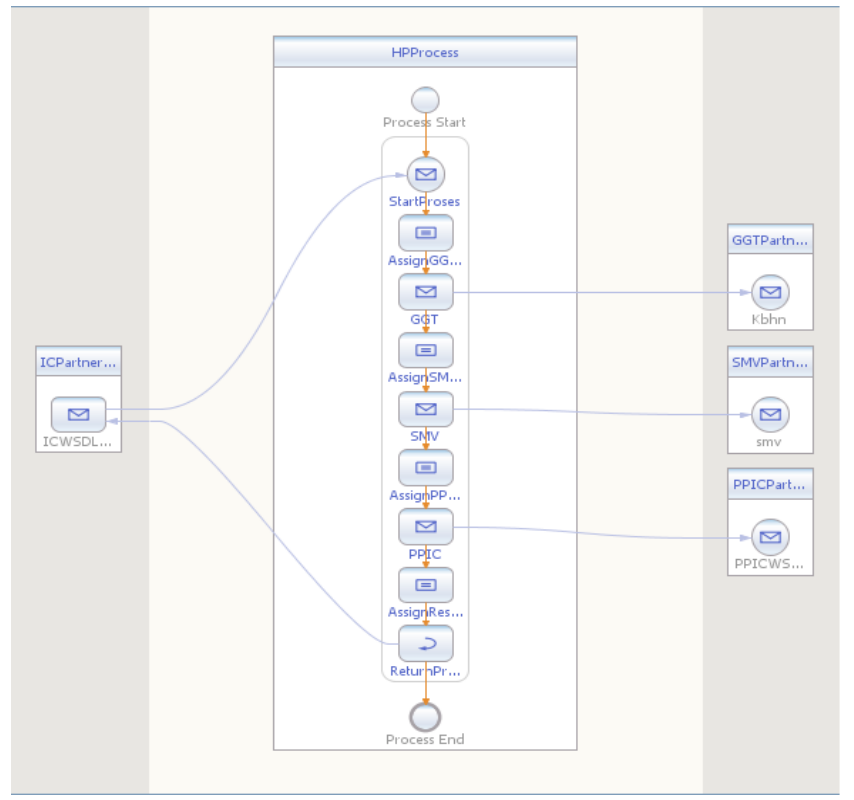

Fig. 10:BPELWorkflow Design 
Component Diagram will be implemented by using ESB Glassfish in the form of composite applications. Each part consists of application within with the use of MySQL database on GGT, SMV and PPIC. The composite application is used to build a set of applications consisting of separated components (sub-assemblies). Each sub assembly wraps the implementation by providing a well-defined public interface. It can be seen in Figure 11.

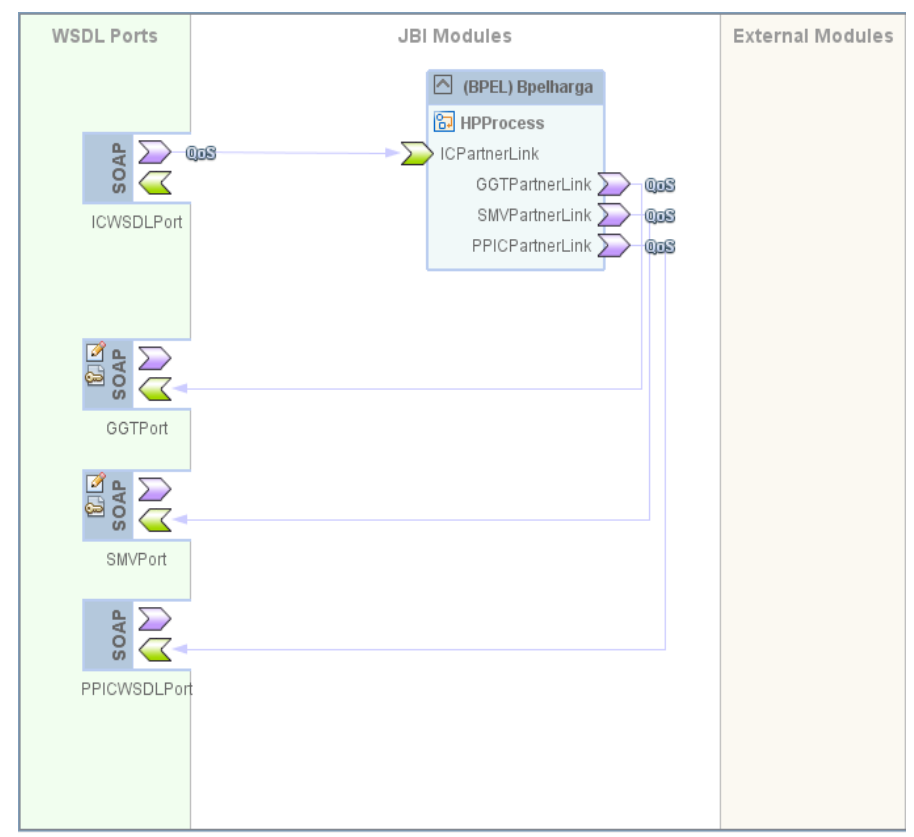

Fig. 11:Design of Composite Application ESB

\section{Test results}

Test results on the PPIC part can be displayed with the composite test results on ESB service. BPEL Orchestration in each section can be determined from the input information of each section, which is used as the basis for determining the cost of materials.

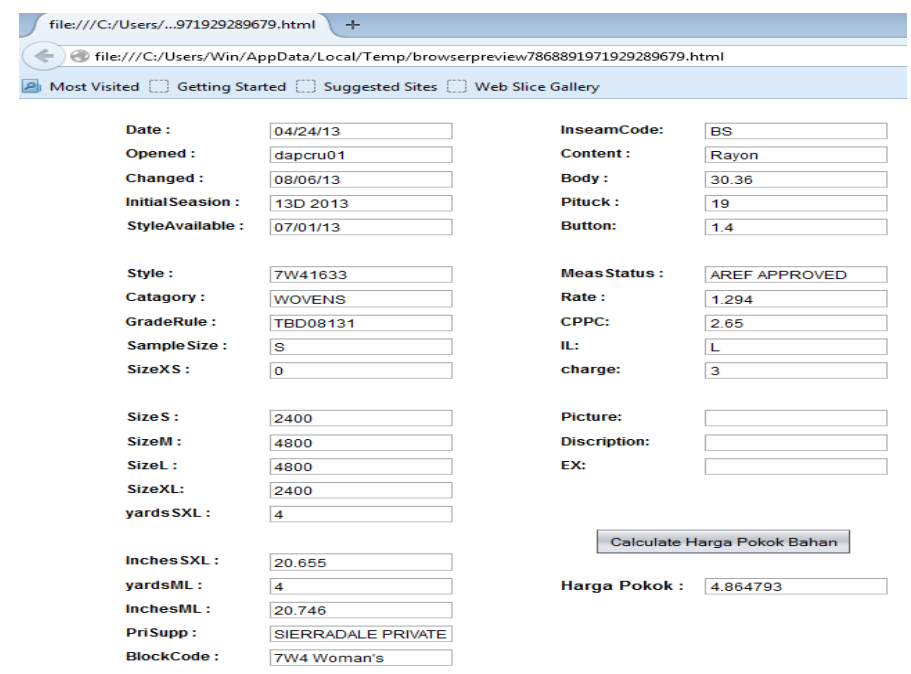

Fig. 12: Application for UI IC

In the process of trial, there are several scenarios to determine the functionality of the program. The trial is performed starting from the entry of the input and process to produce the output. The trial involved test for some major components of this application. In the test results, there are the input process and output process as the results. It can be seen in Figure 13. 


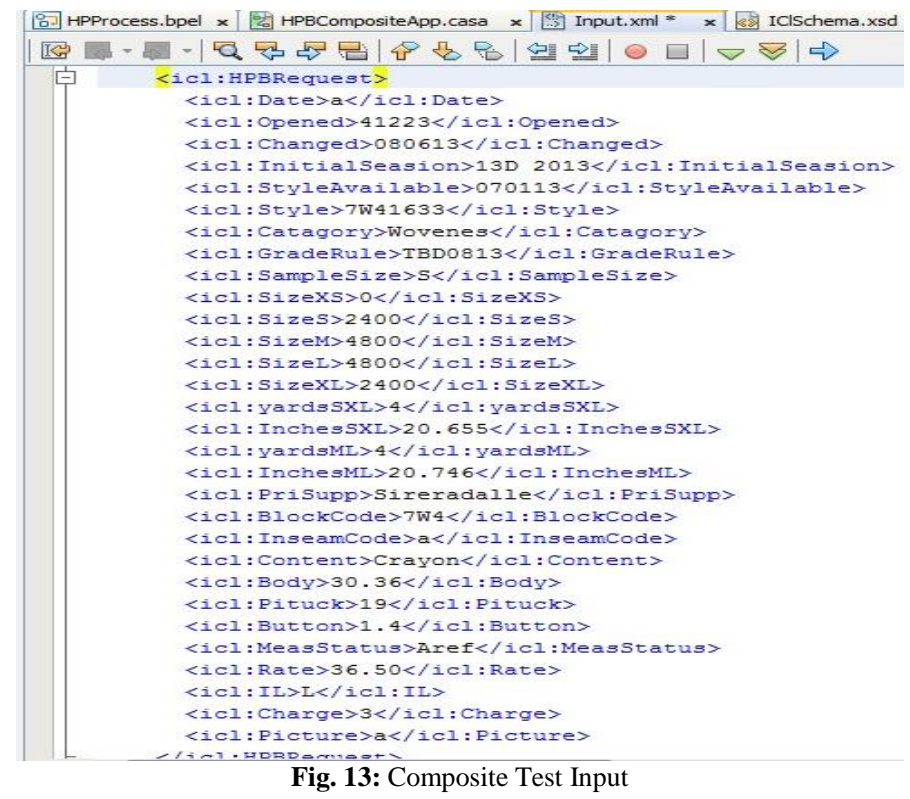

From the observation of analysis, design, implementation and trial stages, the authors conclude as follows:

1) Service Integration by using OpenESB was performed by making a BPEL and a Composite Application for each web service.

2) Web services that are integrated with OpenESB must be stable with no change in operation name, input and output formats for if there is a change, then we require re-creating the BPEL of the web service because OpenESB uses static WSDL.

\section{Discussion}

Based on the analysis and design carried out in the previous chapters, there were SOA based services on the server and information application in each part of the garment as a service user client of this service. The four main service and three main methods that have been generated in the previous chapter will then be tested and integrated. Here are the results of the services implementation:

1) PPIC Service

This PPIC service has a function to display all information related to the cost of materials needs. There were two methods implemented: Displays the cost of materials. (Ns: HMRequest) and: display the PPIC needs. (Get Info)

2) GGT Service

This GGTPPIC service has a function to display all information related to the material needs for each dress. There were two methods implemented: Display the cost of materials. (tns: Kbhn) and: display the GGT needs. (Get Info)

3) IE Service

This IE service has a function to display all information related to the time needs that should be taken for a dress. There were two methods implemented: Display the cost of materials (tns: SMV) and: display the needs of IE. (Get Info)

From the web service results, the next stage was performing service integration design by using BPEL and Composite Application provided by the OpenESB framework. Each BPEL that created consists of a web service that contains a lot of operations, and each BPEL will have a Composite Application. Based on the results that have been performed on a trial and have been produced based on the implementation (Composite Application):

1) Each part got data information that was given in accordance with their needs based on web service, so it no longer got the raw data information directly from the marketing/supplier.

2) The PPIC and marketing department could get the data more efficiently than the services provided from each department with the output of cost of materials.

3) The services provided included calculations required for each department and could directly receive the cost of materials, as a reference for determining the selling price. So the service orchestration application with SOA could give with the need to produce the cost of materials.

\section{Conclusion and recommendations}

In this study, the results suggest some things that have been performed. The integration of several services that come from every part that is PPIC, GGT, IE by using ESB as an integration middleware. So it can be concluded as follows: 
1) In this study, it was successfully built composite web service applications well, to combine the web service that is owned by three applications; web service PPIC from PPIC application, GGT web service from GGT application and IE web service from IE application, so the access of service functions of the IC web service, data distribution services to the service that is needed by each department can be performed through web service composite.

2) Composite service web application service was successfully intercepted on the client side according to the needs of each department well with web-based. The utilization of SOA with ESB in this integration method can also overcome the data distribution based on the services that have been built so it can generate output on each department that needs each other. In particular, the cost of materials that becomes very important data information.

Recommendations from the authors are:

1) In analysis and design, we should use principles of Service Oriented Architecture in order to pay attention on the existing business processes seriously in order to generate completely independent services or a very small interservice dependence.

2) Service that is generated from this study can be developed with further stage of SOA design to produce a service encapsulation.

\section{References}

[1] Erl, T., Service-Oriented Architecture: Concepts, Technology, and Design, Prentice Hall PTR, Upper Saddle River, New Jersey $07458,2005$.

[2] Rifai, Ahmad. "RancangBangunSistemPersediaan (Inventory) Dengan Model As a Service Menggunakan Service Oriented Architecture". IntitutSepuluh November, 2010.

[3] GüntherBudzick, Thomas Dupré, "soa for crm-integration at t-mobile", 2008.

[4] Safuwan, "IntegrasiPerangkatLunak Enterprise Resource Planning (ERP) DenganMenggunakanMetode Service Oriented Architecture (SOA)". IntitutSepuluh November, 2010.

[5] McGovern, J., Sims, O., Jain, A., \& Little, M. Enterprise Service Oriented Architectures: Concepts, Challenges, and Recommendations. Springer Netherlands, 2006.

[6] Andary, J.F. and Sage, A.P., The role of service oriented architectures in systems engineering, Information Knowledge Systems Management 9(2010), IOS Press, 2010.

[7] Erl, T., Service Oriented Architecture: A Field Guide to Integrating XML and Web services, Prentice Hall PTR, Upper Saddle River, New Jersey 07458, 2004

[8] WirantoHeriUtomo, "PemrogramanAplikasiSOA"-Salatiga:FakulatasTeknologiInformasiUKSWPers, 2012.

[9] Juric, M.B., Loganathan, R., Sarang, P., dan Jennings, F., SOA Approach to Integration, Packt Publishing, Birmingham, B27 6PA, UK, 2007.

[10] Erl, T., Service Oriented Architecture: A Field Guide to Integrating XML and Web services, Prentice Hall PTR, Upper Saddle River, New Jersey 07458, 2004.

[11] Luthria, H. And Rabhi, F., Service Oriented Computing in Practice - An Agenda for Research into the Factors Influencing the Organizational Adoption of Service Oriented Architectures, Journal of Theoretical and Applied Electronic Commerce Research, ISSN 0718-1876 Electronic Version VOL 4 / ISSUE 1 / APRIL 2009 / 39-56.

[12] Bieberstein., et al. "Executing SOA: A Practical Guide for The Service Oriented Architecture" IBM Press, Indiana.

[13] Erl, T., Karmarkar, A., Walmsley, P., Haas, H., Yalcina, U., Liu, C.K., Orchard, D., Tost, A., danPasley, J., Web service Contract Design and Versioning for SOA, Prentice Hall PTR, Upper Saddle River, New Jersey 0745, 2008.

[14] Brown Paul C.’ImplementatingSOA: Total Architecture in Practice." Addison Wesely Professional Massachusetts. 2008, 14-18.

[15] Daniel RianoKaparang., Wiranto H. Utomo., Danny Manongga. "Logistics System Design based on Service Oriented Architecture using Thomas Erl Model". Thesis Magister of Information Systems, Satya Wacana Christian University, 2010. 